\title{
The Relationships between Toll-like Receptors and RP215-associated Immunoglobulins Expressed by Cancer Cells \\ Gregory Lee ${ }^{1,2 *}$, Cheng-Yuan Huang ${ }^{1}$, Hao Zhang ${ }^{1,2}$ and Yiting Tang ${ }^{1,2}$
}

${ }^{1}$ UBC Center for Reproductive Health, Vancouver, Canada

${ }^{2}$ Department of Pathology, Shantou University, Shantou, China

\begin{abstract}
Relationships between the expressions of immunoglobulins by cancer cells and those of toll-like receptors were investigated through comparative gene regulation studies to understand their roles and mechanisms of action in cancer immunology. RP215 is a monoclonal antibody generated in 1987 and found to recognize the carbohydrate-associated epitope which is detected preferentially in the heavy chains of immunoglobulins expressed by cancer cells, but not in normal immune cells. In this report, RP215 was used to study interactions of cancerous immunoglobulins with toll-like receptors in the innate immunity of cancer cells. From gene regulation studies, with OC-3-VGH ovarian and C-33A cervical cancer cell lines, it was demonstrated that the expressions of cancerous toll-like receptors (TLR-2, $-3,-4,-6,-7$ and -9 ) are strongly influenced by the incubation of cancer cells with RP215 or antibodies against human IgG. For example, both RP215 and anti-human IgG were found in high correlation to up-regulate TLR-3 expressions by 2.5 and 3.5 fold, respectively, whereas those of TLR-4 and TLR-9 were downregulated by $50 \%$ to $80 \%$ of the untreated. Based on these studies, it is reasonable to postulate that cancerous immunoglobulins are highly involved to modulate the innate immunity of cancer cells to allow the growth/proliferation of cancer cells within the human body.
\end{abstract}

Keywords: RP215; CA215; Cancerous immunoglobulins; Toll-like receptors; Innate immunity

\begin{abstract}
Abbreviations: GAPDH: Glyceraldehyde-3-Phosphate Dehydrogenase; IgG: Immunoglobulin G; IgSF: Immunoglobulin Superfamily; Proteins: Mab Monoclonal Antibody; NFkB-1: Nuclear Factor kappa-B p105 Subunit 1; PAMP: Pathogens-associated Molecular Patterns; PRRs: Pattern Recognition Receptors; RT-PCR: Reverse Transcription Polymerase Chain Reaction; TLR: Toll-like Receptor
\end{abstract}

\section{Background Information}

Since the initial discovery of cancerous immunoglobulins decades ago, a substantial progress has been made regarding their differential expressions from those derived from normal immune cells [1-4]. However, little is known about their roles in growth/regulations of cancer cells as well their molecular mechanisms of action $[5,6]$. These cancerous immunoglobulins differ from those derived from normal immune cells by the presence of a unique carbohydrate-associated epitope recognized by RP215 monoclonal antibody [7]. CA215 is a group of cancer cell-expressed glycoproteins, each of which is attached with RP215-specific epitope and consists mainly of the heavy chain of cancerous immunoglobulins as well as other minor immunoglobulin superfamily proteins, such as T-cell receptors and cell adhesion molecules $[4,8-10]$. Therefore, RP215 may be used to study essential functional roles of cancerous immunoglobulin as well as their interactions with TLRs in innate immunity of cancerous cells [4].

Toll-like receptors are known to play important roles in the host defence from infection and belong to a family of pattern recognition receptors in cancer cells. Since the development of cancer can also be associated with microbial infection, injury, inflammation and tissue repair, the functions of TLRs may be involved in cancer progression and/or carcinogenesis [11-14].

However, it remains to be demonstrated if cancerous immunoglobulins are functionally related to of TLRs in the innate immunity of cancer cells. With RP215 as unique probe for cancerous immunoglobulins, comparative gene regulation studies were performed to investigate interactions or associations between cancerous immunoglobulins or related genes and TLRs in cancer cells. By using semi-quantitative RT-PCR method, results summarized in this review may shed light to our current understanding of cancer immunology which may operate independently of our normal immune system within our body $[15,16]$.

\section{Gene Regulations and Interactions between Cancer Cell- expressed Toll-like Receptors and Immunoglobulins}

Toll-like receptors are best characterized family of pattern recognition receptors (PRRs) which recognize foreign microbes derived from bacteria and/or virus, known in general as pathogens-associated molecular patterns (PAMPS) [17-19]. Activation of TLRs with their ligands may evoke inflammatory response and protect the host from spreading massive pathogens after infection. This is commonly known as innate immune response either in normal or cancer cells. So far, at least nine different TLRs and the corresponding ligands have been identified and expressed among different types of cancer in humans $[11,20,21]$

*Corresponding author: Gregory Lee, UBC Center for Reproductive Health, 9117 Shanghnessy Street, Vancouver, V6P 6R9, Canada, Tel: (778) 322-4651; Fax (604) 325-4677; E-mail: leecyg@gmail.com

Received December 17, 2013; Accepted February 11, 2014; Published February 17,2014

Citation: Lee G, Huang CY, Zhang H, Tang Y (2014) The Relationships between Toll-like Receptors and RP215-associated Immunoglobulins Expressed by Cancer Cells. J Cancer Sci Ther 6: 077-080. doi:10.4172/1948-5956.1000252

Copyright: @ 2014 Lee G, et al. This is an open-access article distributed under the terms of the Creative Commons Attribution License, which permits unrestricted use, distribution, and reproduction in any medium, provided the original author and source are credited. 
Citation: Lee G, Huang CY, Zhang H, Tang Y (2014) The Relationships between Toll-like Receptors and RP215-associated Immunoglobulins Expressed by Cancer Cells. J Cancer Sci Ther 6: 077-080. doi:10.4172/1948-5956.1000252

\section{Strong influence of RP215 and antibodies against cancerous immunoglobulins on gene regulations of toll-like receptors}

Two unrelated cancer cell lines, namely ovarian (OC-3-VGH) and cervical (C-33A) cancer cells were selected for gene regulation studies $[4,16]$. Upon incubation of RP215 or anti-human IgG with either cancer cells, significant up-regulation (50-100\% increase) of NFkB-1 gene was observed. However the effects of those two antibodies on the expressions of immunoglobulins are not as dramatic and consistent $[4,16]$. No significant increase was observed in gene expressions of IgG in the case of OC-3-VGH cancer cells upon incubations of either $\mathrm{RP} 215$ or anti-human IgG. By comparison, up-regulations of IgG were found, with incubation of C-33A cervical cancer cells under the same conditions. The results of this gene regulation analysis are presented and compared in Table 1.

For both OC-3-VGH and C-33A cancer cells, significant and detectable gene expressions of TLRs, including TLR-2, TLR-3, TLR-4, TLR-6, TLR-7 and TLR-9 were observed. However, relative levels of TLR gene expressions vary significantly with the types of cancer cell lines and TLRs [4]. Results of relative expression levels of TLRs in these two types of cancer cells are presented in Figure 1 for comparisons. In the case of OC-3-VGH ovarian cancer cells, TLR-3, TLR-4 and TLR-9 were expressed significantly at higher levels when compared to those by C-33A cervical cancer cells.

On the other hand, C-33A cancer cells were found to express relative high levels of TLR-2, TLR-4 and TLR-9 suitable for further gene regulation and correlation analysis. Upon treatments with RP215 or anti-human IgG, drastic changes in gene expressions of TLRs were observed. Expression levels of selected TLR genes are also presented in Table 1 for comparisons following treatments of two cancer cell lines with either antibody ligand.

In the case of TLR-3 gene, the expression levels were increased upon incubation with RP215 or anti-human IgG by as much as $200 \%$ and $150 \%$, respectively, in OC-3VGH ovarian cancer cells. A similar result was observed for significant up-regulation of TLR-3 gene in C-33A cervical cancer cells upon the antibody treatments. When TLR4 was employed for gene regulation studies, the expression levels were decreased to $10-30 \%$ of the untreated negative control in OC-3-VGH cancer cells, whereas those of C-33A cancer cells were down-regulated to $50-70 \%$ of the control level.

Similarly, down-regulation of TLR-9 gene was commonly observed for both cell lines upon treatments with either antibody added at $10 \mu \mathrm{g} /$ $\mathrm{ml}$ for 24 hour incubation in either of the two cancer cell culture. In the case of C-33A cancer cells, up-regulation of TLR-2 gene was also observed upon antibody treatments.

\section{High correlations between RP215 and anti-immunoglobulins on gene regulations of toll-like receptors}

Both RP215 and anti-human IgG act similarly on gene regulations of selected TLR genes with no exceptions, when two different cancer cell lines were employed for such comparative studies. The results of these gene expression analyses are summarized and presented in Table 1.

From the data presented in Table 1, a correlation analysis was performed to determine the degree of correlation of these two antibody ligands against cancerous immunoglobulins in terms of their respective effect of TLR gene expressions. As presented in Figure 2, a high degree of correlation was obtained when their relative effects on gene regulations are taken into consideration $\left(R^{2}=0.9467\right)$.

In a separate study, a monoclonal antibody against TLR- 4 was used as the probe to study its effects on gene expression of TLR-4 in cancer cells. Similar to the effects of RP215 or anti-human IgG, anti TLR-4 monoclonal antibody also induces significant down-regulation of TLR4 gene by as much as $20-30 \%$ of the control level in either cancer cell line. Anti-TLR-4 Mab was also found to induce significant apoptosis, similar to that observed for RP215 or anti-human IgG $[4,9,22,23]$ Unexpectedly, anti-TLR-4 monoclonal antibody has little or no effect on the gene expressions of IgG in either cancer cells following similar gene regulation analysis.

\begin{tabular}{|c|c|c|c|}
\hline \multirow{3}{*}{ Gene } & \multirow{3}{*}{$\begin{array}{l}\text { Antibody } \\
\text { Ligands }\end{array}$} & \multicolumn{2}{|c|}{ Cancer Cell Line } \\
\hline & & OC-3-VGH & C-33A \\
\hline & & \multicolumn{2}{|c|}{ (Observed percentages of gene expressions ${ }^{a}$ ) } \\
\hline \multirow[t]{2}{*}{$\lg G$} & RP215 & $108 \pm 2^{* \mathrm{~b}, \mathrm{c}}$ & $160 \pm 5$ \\
\hline & Anti-human IgG & $105 \pm 5^{\star c}$ & $166 \pm 4$ \\
\hline \multirow[t]{2}{*}{$\mathrm{NF} \kappa \mathrm{B}-1$} & RP215 & $141 \pm 16$ & $182 \pm 9$ \\
\hline & Anti-human IgG & $153 \pm 10$ & $176 \pm 11$ \\
\hline \multirow[t]{2}{*}{ TLR-3 } & RP215 & $326 \pm 32$ & $329 \pm 12$ \\
\hline & Anti-human IgG & $243 \pm 25$ & $141 \pm 18$ \\
\hline \multirow[t]{2}{*}{ TLR-4 } & RP215 & $12 \pm 9$ & $46 \pm 8$ \\
\hline & Anti-human IgG & $28 \pm 18$ & $71 \pm 6$ \\
\hline \multirow[t]{2}{*}{ TLR-9 } & RP215 & $17 \pm 10$ & $39 \pm 4$ \\
\hline & Anti-human IgG & $43 \pm 8$ & $60 \pm 4$ \\
\hline \multirow[t]{2}{*}{ TLR-2 } & RP215 & & $152 \pm 14$ \\
\hline & Anti-human IgG & & $116 \pm 13$ \\
\hline
\end{tabular}

${ }^{a}$ The gene expressions levels of cancer cells without treatments were adjusted to $100 \%$. (based on comparison with the internal control gene of GAPDH)

${ }^{b}$ Average and standard deviation are presented in each case

${ }^{c}$ All data presented are statistically significant at $\mathrm{P}<0.01$ except those labeled with which are not statistical different from the negative control

Table 1: Effects of RP215 and anti-human IgG on Expressions of Selected Genes from OC-3-VGH ovarian and C-33A cervical cancer cells.

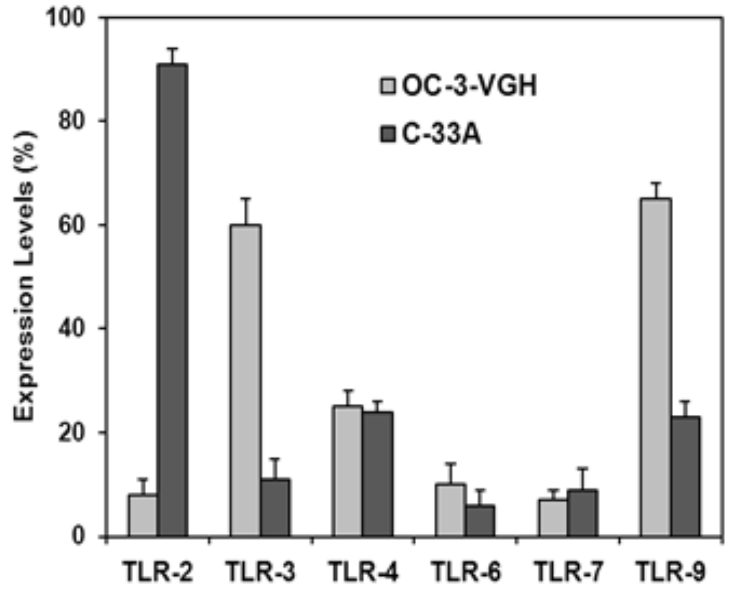

Figure 1: Toll-like receptor (TLR) expression levels of OC-3-VGH ovarian and C-33A cervical cancer cell lines. Semi-quantitative RT-PCR was used to reveal relative expression levels of various toll-like receptors including TLR-2 to TLR-9 from cultured OC-3-VGH ovarian ( $\square$ ) and C-33A cervical ( $)$ cance cells, respectively. Lane 1: TLR-2; Lane 2: TLR-3; Lane 3: TLR-4; Lane 4: TLR 6; Lane 5: TLR-7; Lane 6: TLR-9. Expression of GAPDH gene served as the internal control were adjusted to $100 \%$. Percent expression levels of various TLR genes with respect to that of GAPDH are presented for comparisons. Obtained and modified from [4] with permission. 


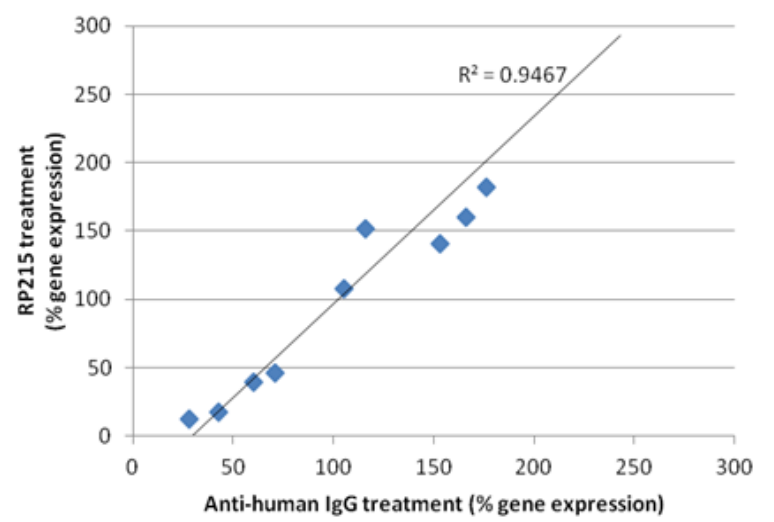

Figure 2: Correlation analysis of gene regulations of IgG, NFKB-1 and selected TLR genes upon separate treatments with RP215 and antihuman IgG in two cancer cell lines. The data $(\bullet)$ represent relative gene expression levels of IgG, NFKB-1, TLR-2, $-3,-4$ and -9 , respectively, in OC3-VGH ovarian and/or C-33A cervical cancer cells upon separate treatments with RP215 and anti-human IgG and are plotted for correlation analysis. The correlation coefficients $\left(R^{2}\right)$ for treatments between these two antibody ligands were determined to be $R^{2}=0.9467$.

\section{Discussion}

Strong unidirectional effects of RP215 or antiimmunoglobulins on gene expressions of toll-like receptors

In this study, efforts have been made to study the effects of RP215 or anti-human IgG on gene regulations of toll-like receptors in the innate immunity of cancer cells. Through gene regulation studies, it was demonstrated that gene expressions of toll-like receptors, especially TLR-3, TLR-4 and TLR-9 in two different cultured cancer cell lines, are strongly influenced by treatments of these two antibody ligands. On the contrary, anti-TLR- 4 monoclonal antibody has no effects on gene expression of IgG in cancer cells. This observation seems to suggest that the controls of selected TLR genes by cancerous immunoglobulins are unidirectional. Since toll-like receptors are required for the innate immune system of cancer cells, the expression of cancerous immunoglobulins may in part, explain their requirements for the growth/survival of cancer cells [12,24-28].

\section{NFKB-1 is a key gene regulator}

It is interesting to note that NFkB-1 was significantly up-regulated by treatments either with RP2 15 or antibodies against human $\operatorname{IgG}$ in either cancer cells $[4,16]$. Since NFKB is a transcription factor involved in the activation or expression of as many as 200 genes important for biological functions of various cell types [11,12,20,21,29-32]. Therefore, the associations between cancerous immunoglobulins and toll-like receptors could be highly regulated by NFkB as a common transcription factor [29-32].

Through gene regulation studies, the biosimilarity between RP215 and anti-human IgG was clearly established with respect to their effects on gene regulations of toll-like receptors in cancer cells (Figure 2).

Strong involvements and mutual interactions of cancerous immunoglobulins and toll-like receptors in cancinogenesis of cancer cells

The involvement of TLRs in the carcinogenesis of cancer cells has been extensively investigated $[11,12,20,21]$. TLRs are known for their abilities to recognize different molecules associated to pathogens, for example lipopolysaccharide (LPS), flagellin, or double strand RNAs $[11,12]$. TLR-related research has greatly advanced recently and shows that TLRs may also play roles in driving tumorigenesis through stimulation, promoting cancer growth and helping the surveillance of cancer cells against the host immune system [11,12,20,21]. Understanding the mechanism and signaling pathway promises potential therapies against infection, inflammation as well as cancer $[11,12,20,21]$.

We believe that endogeneous antigens may be present and hostile to tumor cells in our normal body environment. Cancerous immunoglobulins are required to neutralize these antigens for the protection of cancer cells as additional defense mechanism for the survival and proliferation of cancer cells, similar to those of toll-like receptors in the innate immunity system against foreign pathogens. Therefore, strong interactions of these two systems may have to operate simultaneously with strong interactions.

\section{Clinical significance of cancerous immunoglobulins and toll- like receptors in cancer immunology}

Based on results of the present studies, it seems reasonable to suggest that TLRs and cancerous immunoglobulins may play similar or parallel roles in neutralizing undesirable "antigen/pathogen" in the innate immunity of cancer cells [33,34]. Both could play equally important roles for the immune protections in the immune system of cancer cells. Strong interactions between cancerous immunoglobulins and toll-like receptor through a common regulator, or transcription factor such as NFkB cannot be underestimated. This may be one of the most important mechanisms of action for the development of RP215 as an anti-cancer drug during cancer immunotherapy in humans.

\section{Acknowledgements}

This research project was supported in parts by NRC-IRAP program (\#794354) of Canada. Helpful discussion on this topic with Suefay Liu is acknowledged.

\section{Conflict of Interest}

Gregory Lee is co-founder of Vancouver Biotech Ltd. The other two have nothing to declare.

\section{References}

1. Kimoto $Y$ (1998) Expression of heavy-chain constant region of immunoglobulin and T-cell receptor gene transcripts in human non-hematopoietic tumor cell lines. Genes Chromosomes Cancer 22: 83-86.

2. Qiu X, Zhu X, Zhang L, Mao Y, Zhang J, et al. (2003) Human epithelial cancers secrete immunoglobulin $\mathrm{g}$ with unidentified specificity to promote growth and survival of tumor cells. Cancer Res 63: 6488-6495.

3. Li M, Feng DY, Ren W, Zheng L, Zheng H, et al. (2004) Expression of immunoglobulin kappa light chain constant region in abnormal human cervical epithelial cells. Int J Biochem Cell Biol 36: 2250-2257.

4. Tang $\mathrm{Y}$, Zhang $\mathrm{H}$, Lee G. (2013) Similar gene regulation patterns for growth inhibition of cancer cells by RP215 or anti-antigen receptors. J Cancer Sci Ther 5: $200-208$.

5. Babbage G, Ottensmeier CH, Blaydes J, Stevenson FK, Sahota SS (2006) Immunoglobulin heavy chain locus events and expression of activation-induced cytidine deaminase in epithelial breast cancer cell lines. Cancer Res 66: $3996-$ 4000.

6. Chen Z, Gu J (2007) Immunoglobulin G expression in carcinomas and cancer cell lines. FASEB J 21: 2931-2938.

7. Lee G, Laflamme E, Chien $\mathrm{CH}$, Ting $\mathrm{HH}$ (2008) Molecular identity of a pan cancer marker, CA215. Cancer Biol Ther 7: 2007-2014.

8. Lee G, Ge B (2009) Cancer cell expressions of immunoglobulin heavy chains with unique carbohydrate-associated biomarker. Cancer Biomark 5: 177-188. 
Citation: Lee G, Huang CY, Zhang H, Tang Y (2014) The Relationships between Toll-like Receptors and RP215-associated Immunoglobulins Expressed by Cancer Cells. J Cancer Sci Ther 6: 077-080. doi:10.4172/1948-5956.1000252

9. Lee G, Zhu M, Ge B, Potzold S (2012) Widespread expressions of immunoglobulin superfamily proteins in cancer cells. Cancer Immunol Immunother 61: 89-99.

10. Lee G, Cheung AP, Li B, Ge B, Chow PM (2012) Molecular and immunocharacteristics of immunoglobulin-like glycoproteins in cancer cell-expressed biomarker, CA215. Immunol Invest 41: 429-446.

11. Rakoff-Nahoum S, Medzhitov R (2009) Toll-like receptors and cancer. Nat Rev Cancer 9: 57-63.

12. So EY, Ouchi T (2010) The application of Toll like receptors for cancer therapy. Int J Biol Sci 6: 675-681.

13. Muccioli M, Sprague L, Nandigam H, Pate M, Benencia F (2012) Toll-like receptors as novel therapeutic targets for ovarian cancer. ISRN Oncol 2012: 642141.

14. Pradere JP, Dapito DH, Schwabe RF (2013) The Yin and Yang of Toll-like receptors in cancer. Oncogene.

15. Lee G (2012) Cancerous immunoglobulins and CA215: implications in cancer immunology. Am J Immunol 8: 101-116.

16. Lee G, Liu S (2013) Roles of antigen receptors and CA215 in the innate immunity of cancer cells. Open J Immunol 3: 127-138.

17. Takeda K, Kaisho T, Akira S (2003) Toll-like receptors. Annu Rev Immunol 21: 335-376.

18. Medzhitov R, Janeway CA Jr (2002) Decoding the patterns of self and nonself by the innate immune system. Science 296: $298-300$.

19. Takeda K, Akira S (2005) Toll-like receptors in innate immunity. Int Immunol 17: $1-14$.

20. O Neill LAJ (2008) Toll-like receptors in cancer. Oncogene 27: 158-160.

21. O'Neill LA, Bryant CE, Doyle SL (2009) Therapeutic targeting of Toll-like receptors for infectious and inflammatory diseases and cancer. Pharmacol Rev 61: 177-197.

22. Lee G, Cheung AP, Ge B, Zhu M, Giolma B, et al. (2012) CA215 and GnRH receptor as targets for cancer therapy. Cancer Immunol Immunother 61: 18051817
23. Lee G, Azadi P (2012) Peptide mapping and glycoanalysis of cancer cellexpressed glycoproteins CA215 recognized by RP215 monoclonal antibody. J Carbohydr Chem 31: 10-30.

24. Li M, Zheng H, Duan Z, Liu H, Hu D, et al. (2012) Promotion of cell proliferation and inhibition of ADCC by cancerous immunoglobulin expressed in cancer cell lines. Cell Mol Immunol 9: 54-61.

25. Vesely MD, Kershaw MH, Schreiber RD, Smyth MJ (2011) Natural innate and adaptive immunity to cancer. Annu Rev Immunol 29: 235-271.

26. Schmausser B, Andrulis M, Endrich S, Müller-Hermelink HK, Eck M (2005) Tolllike receptors TLR4, TLR5 and TLR9 on gastric carcinoma cells: an implication for interaction with Helicobacter pylori. Int J Med Microbiol 295: 179-185.

27. Furrie E, Macfarlane S, Thomson G, Macfarlane GT; Microbiology \& Gut Biology Group; Tayside Tissue \& Tumour Bank (2005) Toll-like receptors-2, -3 and -4 expression patterns on human colon and their regulation by mucosalassociated bacteria. Immunology 115: 565-574.

28. Huang B, Zhao J, Unkeless JC, Feng ZH, Xiong H (2008) TLR signaling by tumor and immune cells: A double-edged sword. Oncogene 27: 218-224.

29. Li Q, Withoff S, Verma IM (2005) Inflammation-associated cancer: NF-kappaB is the lynchpin. Trends Immunol 26: 318-325.

30. Griffin JD (2001) Leukemia stem cells and constitutive activation of NF-kappaB Blood 98: 2291.

31. Xie W, Wang $Y$, Huang $Y$, Yang H, Wang J, et al. (2009) Toll-like receptor 2 mediates invasion via activating NF-kappaB in MDA-MB-231 breast cancer cells. Biochem Biophys Res Commun 379: 1027-1032.

32. Pikarsky E, Porat RM, Stein I, Abramovitch R, Amit S, et al. (2004) NF-kappaB functions as a tumour promoter in inflammation-associated cancer. Nature 431 461-466.

33. O'Neill LA (2004) TLRs: Professor Mechnikov, sit on your hat. Trends Immuno 25: 687-693.

34. Lee G, Huang CY, Liu S, Zhang H (2013) The Immunology of Cancer Cells Symbiosis Open Journal Immunology in press. 